\title{
The Organization of the Genetic Code
}

This paper was downloaded from TechRxiv (https://www.techrxiv.org).

\section{LICENSE}

CC BY 4.0

SUBMISSION DATE / POSTED DATE

23-02-2022 / 04-03-2022

\section{CITATION}

Kak, Subhash (2022): The Organization of the Genetic Code. TechRxiv. Preprint. https://doi.org/10.36227/techrxiv.19224741.v1

$\mathrm{DOI}$ 


\title{
The Organization of the Genetic Code
}

\author{
Subhash Kak \\ Chapman University, Orange, CA 92866 \& \\ Oklahoma State University, Stillwater, OK 74078, USA \\ Email: subhash.kak@okstate.edu
}

\begin{abstract}
The genetic code has two puzzling anomalies: first, when viewed as 64 sub-cubes of a $4 \times 4 \times 4$ cube, the codons for serine (S) are not contiguous; and there are amino acid codons with zero redundancy, which goes counter to the objective of error-correction. To make sense of this, the paper shows that the genetic code must be viewed not only on stereochemical, coevolution, and error-correction considerations, but also on two additional factors of significance to natural systems, that of an information-theoretic dimensionality of the code data, which requires that the optimum natural code, as opposed to an engineered one, be characterized by $e$-dimensionality, and the principle of maximum entropy. One implication of noninteger dimensionality is self-similarity to different scales, and it is shown that the genetic code does satisfy this property, and it is further shown that the maximum entropy principle operates through the scrambling of the elements in the sense of maximal algorithmic information complexity. These two additional considerations explain several aspects of its organization.
\end{abstract}

\section{Introduction}

All life forms on earth share the same genetic code, with some small variations. This standard code is a mapping of 64 codons onto a set of 20 amino acids and the stop signal. The main features of the genetic code (Figure 1) are that XYU and $\mathrm{XYC}$ always code the same amino acid; XYA and XYG also code the same amino acid, with two exceptions of AUG and UGG; and in half the cases $\mathrm{XY}+$ represent a single amino acid, and each of the following cases $\mathrm{CC}+, \mathrm{CG}+, \mathrm{GC}+$, and $\mathrm{GG}+$ map to the same amino acid.

A uniform coding will require just about 3 codons for each amino acid but the groups range from 1 to 6 . According to one theory, this non-uniformity arises from stereochemical reasons [1-7] related to physico-chemical affinity between amino 
acids and the cognate codons (anticodons). Another theory sees the universality of the code structure having coevolved with amino acid biosynthesis pathways [811]. To make sense of this, the paper shows that the genetic code must be viewed not only on stereochemical, coevolution, and error-correction considerations, but also on two additional factors of significance to natural systems, that of an information-theoretic dimensionality of the code data [12-14], which requires that the optimum natural code, as opposed to an engineered one, be characterized by $e$ dimensionality, and the principle of maximum entropy [15]. The coevolution argument can also be seen in terms of complexity theory in that it represents the bottom of an attraction basin [16] for which the cost of climbing out is prohibitively high.

The error minimization theory views the minimization of the adverse effect of mutations and translation errors as principal factors in the code's evolution [1722]. Analysis of the structure shows that the code is robust to translational errors, but there are other codes that are equally robust or even better [23], and so it could have evolved from a random code and then frozen into its current from due to coevolution.

\begin{tabular}{|c|c|c|c|c|c|c|c|}
\hline UUU & \multirow{2}{*}{$\mathbf{F}$} & UCU & \multirow{4}{*}{$\mathbf{S}$} & UAU & \multirow{2}{*}{$\mathbf{Y}$} & UGU & \multirow{2}{*}{ C } \\
\hline UUC & & UCC & & UAC & & UGC & \\
\hline UUA & \multirow{2}{*}{$\mathbf{L}$} & UCA & & UAA & \multirow{2}{*}{ Stop } & UGA & Stop \\
\hline UUG & & UCG & & UAG & & UGG & W \\
\hline CUU & \multirow{4}{*}{$\mathbf{L}$} & $\mathrm{CCU}$ & \multirow{4}{*}{$\mathbf{P}$} & CAU & \multirow{2}{*}{ H } & CGU & \multirow{4}{*}{$\mathbf{R}$} \\
\hline CUC & & CCC & & CAC & & CGC & \\
\hline CUA & & CCA & & CAA & & CGA & \\
\hline CUG & & CCG & & CAG & $\mathbf{Q}$ & CGG & \\
\hline AUU & & $\mathbf{A C U}$ & \multirow{4}{*}{$\mathbf{T}$} & AAU & \multirow{2}{*}{$\mathbf{N}$} & $\mathbf{A G U}$ & \multirow{2}{*}{$\mathbf{S}$} \\
\hline AUC & & $\mathbf{A C C}$ & & AAC & & $\mathbf{A G C}$ & \\
\hline AUA & & ACA & & AAA & \multirow{2}{*}{$\mathbf{K}$} & AGA & \multirow{2}{*}{$\mathbf{R}$} \\
\hline AUG & I & ACG & & AAG & & AGG & \\
\hline GUU & \multirow{4}{*}{ V } & GCU & \multirow{4}{*}{ A } & GAU & \multirow{2}{*}{ D } & GGU & \multirow{4}{*}{ G } \\
\hline GUC & & GCC & & GAC & & GGC & \\
\hline GUA & & GCA & & GAA & $\mathbf{F}$ & GGA & \\
\hline GUG & & GCG & & GAG & $\mathbf{E}$ & GGG & \\
\hline
\end{tabular}

Figure 1. The standard genetic code

It has not been pointed out before, but the algebraic theory of error minimization seen in a three-dimensional data space needs to be modified for natural systems. While it is appropriate for engineered codes that are used in computer science, it is not true of natural codes because the data space in natural systems is not 3- 
dimensional, but $e$-dimensional. Furthermore, the evolution of the code must proceed according to the maximal entropy principle [24] according to which of all the distributions that satisfy the constraints associated with the problem, the one with the largest entropy should be chosen.

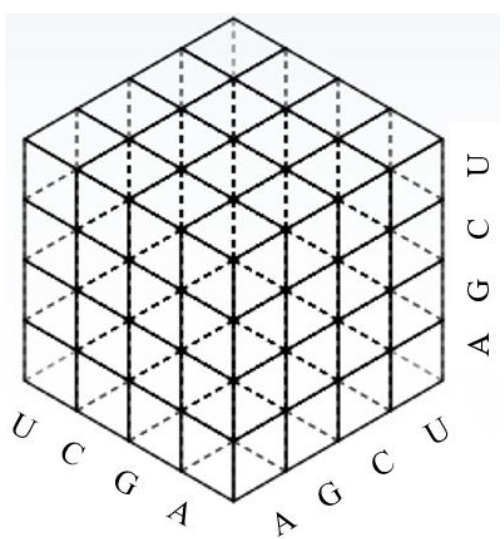

Figure 2. Anomalously, codon groups for serine in the $4 \times 4 \times 4$ codon cube are not contiguous

The proof of the assertion that $e$-dimensionality is optimal is elementary. Each coordinate axis in the general abstract space may be viewed as a bin. Assume a total of $d$ bins and label them as $1,2,3 \ldots d$. The utilization of the system would be optimal if each of the bins carries the same information or the probability of the use of each is equal to $1 / d$. The information associated with each bin then equals $\ln d$.

This information increases as $d$ increases, but this increase is obtained at the cost of the use of the larger and thus more expensive bin set. The information efficiency per bin is: $E(d)=\frac{\ln d}{d}$. Its maximum value is obtained by taking the derivative of $E(d)$ and equating that to zero, which yields $d_{\mathrm{opt}}=e$. This approach leads to the structure of several fractal solutions to natural systems [24-26].

This paper presents a solution of the problem of the non-uniform distribution of codon groups from an overarching information theoretic perspective considering the $e$-dimensional organization of data and application of the maximal entropy principle. It is shown that the information associated with the genetic code reflects self-similarity across different scales that may be seen as a power law or an approximate Virahānika-Fibonacci (VF) mapping. It is shown that the group data is scrambled in a maximum entropy sense. 


\section{Non-uniformity of the code}

Figure 3 summarizes the non-uniformity of groups in the genetic code, where A represents a single codon with two of such type, B represents two codons in each group and 9 represents the number of such type, $C$ represents three codons in each group and there is a single one of this type, D represents 4 codons that each map into a amino acid and there are five of this kind, there is no set of 5, and F represents 6 codons in the group and there are three of this kind.

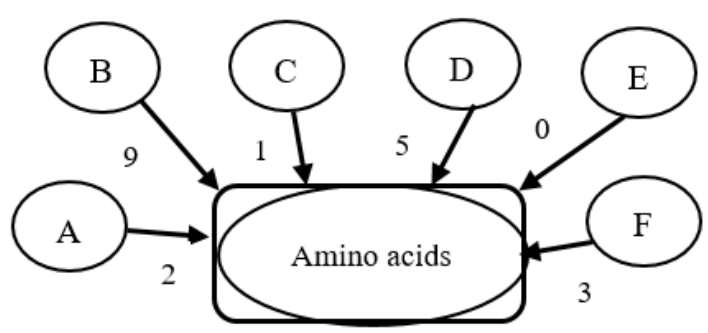

Figure 3. Codons to amino acids as different groups; the numbers represent the members of the group

Given that the uniform coding requires three codons, perhaps a range from 2 to 4 could have been understood on stereochemical grounds, but why should the code have groups that range from 1 to 6 , where the ones corresponding to methionine and tryptophan, each of which is encoded by a single codon don't have any redundancy at all? The fact that the redundancy of the coding varies in an extreme manner indicates that none of the theories are entirely satisfactory, and one needs to find the overarching principle behind the code.

\section{Data dimensionality optimization as overarching principle}

For the case of limited data, it was recently also shown that the randomness and self-similarity can be captured by data amplification [27], allowing one to determine whether the distribution in Table 1 is close to values given by the first digit phenomenon, which is scale invariant.

Table 1. Number of codons to amino acid (derived from Figure 1)

\begin{tabular}{|l|l|l|l|l|l|l|}
\hline Type name & A & B & C & D & F & G \\
\hline codons to amino acid, $k$ & 1 & 2 & 3 & 4 & 5 & 6 \\
\hline number of this type, $f(k)$ & 2 & 9 & 1 & 5 & 0 & 3 \\
\hline Codon count & 2 & 18 & 3 & 20 & 0 & 18 \\
\hline
\end{tabular}


Certain stereochemical considerations require that 3 of the 64 codons be assigned to STOP; this leaves us with 61 codons that should be mapped to the 20 amino acids (see the $e$-dimensionality basis of this number [28]) in such a manner that self-similarity is maintained in the mapping.

If the number of codons assigned to a specific amino acid is $k$, and the number of this type is $f(k)$, where $\mathrm{k}$ ranges from 1 to $\mathrm{n}, \mathrm{n}<20$, and $f(\mathrm{k})$ is an integer greater than or equal to zero, then

$$
\begin{aligned}
& f(k)=(2,9,1,5,0,3) \\
& \sum_{k=1}^{n} k f(k)=61
\end{aligned}
$$

and since the number of amino acids is 20 , we have:

$$
\sum_{k=1}^{n} f(k)=20
$$

We can write the solution $f(\mathrm{k})$ vector as (a, b, c, d, e, f). With just these two constraints there will be many solutions. But there is an additional constraint that needs to be considered. This is the requirement of self-similarity, which is a consequence of noninteger dimensionality. Self-similarity emerges out of recursion and this may be seen as below:

$$
f(k)=g(f(k-1), f(k-2))
$$

As shown recently [27], the application of the self-similarity constraint for sparse data can be done using the first digit phenomenon after amplifying the data by multiplying it by non-occurring numbers in the original data set. The values $a, b$, $\mathrm{c}, \mathrm{d}, \mathrm{e}, \mathrm{f}$ were enhanced by multiplying them with different $\alpha$ 's that are absent in the original set. It was shown that the genetic code sequence numbers did satisfy the first digit phenomena numbers well after the data was amplified. This provided a justification of the randomness of the data.

\section{Maximization of entropy}

Let us now investigate the recursive function at the basis of the data according to (3). It may be assumed that randomness inherent in the generation process effectively scrambles the data but for the sake of convenience this may be split into a 
two-part process. Thus, we will consider entropy maximization as a distinct operation, although in nature this is bound to be entangled with the underlying process that generates self-similarity.

In algorithmic information theory, the Kolmogorov complexity of an object [29], such as a piece of text, is the length of the shortest program, in some programming language that produces the object as output (parenthetically, it is also of relevance in the study of the phenomenon of consciousness [30]). It is a measure of the information needed to specify the object. For permutations, we propose that full cycle permutations that are not linear represent maximal entropy. This distribution must encode for critical biological information related to robustness, selection and evolvability.

In the algorithmic complexity theory perspective, it is known that powers of a primitive root modulo prime $p$ is optimal because it leads to a full polynomial in determining the inverse [31]. This is the reason that powers of primitive roots are used in cryptography protocols for the complexity of inverting them makes brute force prohibitively expensive. If $1,2, . . p-1$ transforms into $g(1), g(2), \ldots g(p-$ $1)$, then the permutation is viewed as $g(1) \rightarrow g(2), g(2) \rightarrow g(3), \ldots g(p-2) \rightarrow$ $g(p-1)$

In the genetic code, we have 6 objects (groups of codons), therefore, we can consider the primitive root modulo 7, and it has two such roots: 3 and 5. The two maximum information scrambling transformation of the numbers 123456 will be as $3^{i} \bmod 7$ or $5^{i} \bmod 7, i=1,2, \ldots 6$. These sequences will be taken to be the successive states in the mapping.

The first one of these creates the sequence R: 326451 and the second one yields S: 546231 . The first represents the permutation in which $3 \rightarrow 2 ; 2 \rightarrow$ $6 ; 6 \rightarrow 4 ; 4 \rightarrow 5 ; 5 \rightarrow 1 ; 1 \rightarrow 3$, whereas the second one is the inverse of it: $5 \rightarrow$ $4 ; 4 \rightarrow 6 ; 6 \rightarrow 2 ; 2 \rightarrow 3 ; 3 \rightarrow 1 ; 1 \rightarrow 5$. The permutations $\mathrm{R}$ and $\mathrm{S}$ may be written in the two-line form:

$$
R:\left(\begin{array}{llllll}
1 & 2 & 3 & 4 & 5 & 6 \\
3 & 6 & 2 & 5 & 1 & 4
\end{array}\right) ; \quad S:\left(\begin{array}{llllll}
1 & 2 & 3 & 4 & 5 & 6 \\
5 & 3 & 1 & 6 & 4 & 2
\end{array}\right)
$$

Let the number count associated with the codon groups of the genetic code (2 91 503 ) be written below these permutations: 


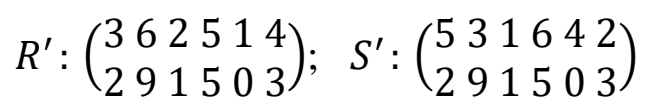

If $k^{\prime}$ is relabeled to 123456 , then we get the following assignments by simply reading off the numbers in sequence in the two-line representation above. Thus 1 $\rightarrow 0 ; 2 \rightarrow 1 ; 3 \rightarrow 2 ; 4 \rightarrow 3 ; 5 \rightarrow 5 ; 6 \rightarrow 9$, for $R^{\prime \prime}$, and $1 \rightarrow 1 ; 2 \rightarrow 3 ; 3 \rightarrow 9 ; 4 \rightarrow$ $0 ; 5 \rightarrow 2 ; 6 \rightarrow 5$, for $S^{\prime \prime}$, and

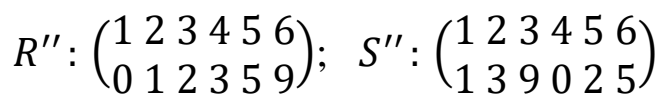

A straightforward analysis shows that the scrambling $S^{\prime \prime}$ leads to a mapping that violates the constraint (2), and so it may be ignored.

The permutation $R^{\prime \prime}$ lends itself to the recursive formula underlying the Virahānka-Fibonacci (VF) series [32-34]:

$$
f\left(k_{m}^{\prime}\right)=f\left(k_{m-1}^{\prime}\right)+f\left(k_{m-2}^{\prime}\right)
$$

It is different from the VF series by a count of 1 at the first and the last location that may be seen by comparing 012359 to 112358 . The deviation of it at the first and last places may be ascribed to the finiteness of the data set.

The $f\left(k^{\prime}\right)$ values may be seen as an approximate power law series for the last three places:

$1 \times 2=2$, and then:

$$
\begin{aligned}
& 2 \times 2-1=3, \\
& 2 \times 3-1=5, \\
& 2 \times 5-1=9
\end{aligned}
$$

The number of each type is also in a broad distribution with values ranging from 0 to 9 , with the rank order frequencies of $9,5,3,2,1,0$ which we have shown earlier [27] is consistent with the Zipf distribution. In other words, the code data 
corresponds to an optimality principle in communications, minimizing a combination of the "information-theoretic communication inefficiency and direct signal cost" [35].

\section{Conclusions}

The paper explained two significant anomalies, related to non-uniform redundancy and mapping of an amino acid into non-contiguous regions, in the organization of the genetic code. It showed that the code must be viewed not only on stereochemical, coevolution, and error-correction considerations, but also on two additional factors of significance to natural systems: information-theoretic dimensionality, and maximal data entropy. It was shown that the genetic code satisfies the self-similarity property, and it undergoes scrambling generated by a maximum length algebraic transformation consistent with a process of entropy maximization.

The larger implications of this study is that two-dimensional and threedimensional discrete patterns in natural systems must also be studied not only in relation to the underlying recursive transformations but also for the maximization of entropy.

\section{References}

1. Crick, F.H.C. 1968. The origin of the genetic code. J. Mol. Biol. 38:36779

2. Di Giulio, M. 2005. The origin of the genetic code: theories and their relationships, a review. Biosystems 80:175-84

3. Goodarzi, H., Nejad, H.A., Torabi, N. 2004. On the optimality of the genetic code, with the consideration of termination codons. Biosystems 77:163-73

4. Novozhilov, A. S., Wolf, Y. I., \& Koonin, E. V. 2007. Evolution of the genetic code: partial optimization of a random code for robustness to translation error in a rugged fitness landscape. Biology direct, 2, 24.

5. Grosjean, H., Westhof, E. 2016. An integrated, structure- and energybased view of the genetic code. Nucleic Acids Res. 44:8020-40

6. Koonin, E. V. 2017. Frozen accident pushing 50: Stereochemistry, expansion, and chance in the evolution of the genetic code. Life 7:E22

7. Sella, G., Ardell, D.H. 2006. The coevolution of genes and genetic codes: Crick's frozen accident revisited. J. Mol. Evol. 63:297-313 
8. Francis, B.R. 2013. Evolution of the genetic code by incorporation of amino acids that improved or changed protein function. J. Mol. Evol. 77:134-58

9. Sengupta, S., Aggarwal, N., Bandhu, A.V. 2014. Two perspectives on the origin of the standard genetic code. Orig. Life Evol. Biosph. 44:287-91

10. Di Giulio, M. 2016. The lack of foundation in the mechanism on which are based the physico-chemical theories for the origin of the genetic code is counterposed to the credible and natural mechanism suggested by the coevolution theory. J. Theor. Biol. 399:134-40

11. Firnberg, E, Ostermeier, M. 2013. The genetic code constrains yet facilitates Darwinian evolution. Nucleic Acids Res. 41:7420-28

12. Kak, S. 2020. Information theory and dimensionality of space. Scientific Reports 10, 20733. https://www.nature.com/articles/s41598-020-77855-9

13. Kak, S. 2021. Asymptotic freedom in noninteger spaces. Scientific Reports 11, 1-5. https://www.nature.com/articles/s41598-021-83002-9

14. Kak, S. 2021. The intrinsic dimensionality of data. Circuits Syst. Signal Process. 40, 2599-2607 (2021); https://doi.org/10.1007/s00034-02001583-8

15. De Martino, A., \& De Martino, D. 2018. An introduction to the maximum entropy approach and its application to inference problems in biology. Heliyon, 4(4), e00596; https://doi.org/10.1016/j.heliyon.2018.e00596

16. Jaynes, E. T. 2003. Probability Theory: The Logic of Science. Cambridge University Press.

17. Kak, S. 2022. Number of autonomous cognitive agents in a neural network. Journal of Artificial Intelligence and Consciousness.

18. Jestin, J.L., Kempf, A. 2009. Optimization models and the structure of the genetic code. J. Mol. Evol. 69:452-57

19. Lobanov, A.V., Turanov, A.A., Hatfield, D.L., Gladyshev, V.N. 2010. Dual functions of codons in the genetic code. Crit. Rev. Biochem. Mol. Biol. 45:257-65

20. Massey, S.E. 2008. A neutral origin for error minimization in the genetic code. J. Mol. Evol. 67:510-16

21. Buhrman, H., van der Gulik, P.T., Kelk, S.M., Koolen, W.M., Stougie, L. 2011. Some mathematical refinements concerning error minimization in the genetic code. IEEE/ACM Trans. Comput. Biol. Bioinform. 8:1358-72

22. Massey, S.E. 2015. Genetic code evolution reveals the neutral emergence of mutational robustness, and information as an evolutionary constraint. Life 5:1301-32 
23. Massey, S.E. 2016. The neutral emergence of error minimized genetic codes superior to the standard genetic code. J. Theor. Biol. 408:237-42

24. Mandelbrot, B. B. 1983. The Fractal Geometry of Nature. W. H. Freeman

25. Kak, S. 2021. Fractals with optimal information dimension. Circuits Syst. Signal Process. 40, 5733-5743.

26. Kak, S. 2021. New classes of regular symmetric fractals. Circuits Syst. Signal Process. 2022; https://link.springer.com/article/10.1007/s00034022-01966-Z

27. Kak, S. 2022. The iterated Newcomb-Benford distribution for structured systems. Int. J. Appl. Comput. Math 8, 51; https://doi.org/10.1007/s40819-022-01251-2

28. Kak, S. 2021. The e-dimensionality of genetic information. TechRxiv; https://doi.org/10.36227/techrxiv.14977479.v1

29. Li, M. and Vitányi, P. 2009. An Introduction to Kolmogorov Complexity and Its Applications. Springer.

30. Kak, S. 2022. The limits to machine consciousness. Journal of Artificial Intelligence and Consciousness 9, 59-72.

31. Wells, A. 1984. A polynomial form for logarithms modulo a prime. IEEE Transactions on Information Theory, 30, $845-846$

32. Singh, P., 1985. The so-called Fibonacci numbers in ancient and medieval India. Historia Mathematica 12: 229 - 244

33. Knuth, D., 2006. The Art of Computer Programming, Generating All Trees History of Combinatorial Generation. Addison-Wesley.

34. Kak, S. 2022. The Virahānka-Fibonacci and related sequences. https://www.academia.edu/71027275/The Virah\%C4\%81\%E1\%B9\%85 $\mathrm{ka}$ and Related Sequences

35. Salge, C., Ay, N., Polani, D., Prokopenko, M. 2015. Zipf's law: balancing signal usage cost and communication efficiency. PLoS ONE 10(10): e 0139475 